\title{
Autocorrelation, Return Horizons, and Momentum in Stock Returns*
}

\author{
Ming-Shiun Pan \\ Department of Finance and Information Management \& Analysis \\ Shippensburg University \\ Shippensburg, PA 17257 \\ Phone: 717-477-1683 \\ E-mail: mspan@ship.edu
}

First version: August 2004

This version: July 2006

${ }^{*}$ I would like thank participants at the 2004 FMA annual meeting for useful comments. 


\title{
Autocorrelation, Return Horizons, and Momentum in Stock Returns
}

\begin{abstract}
In this study we examine Lewellen's (2002) claim that momentum in stock returns is not due to positive autocorrelation as behavioral models suggest. Using portfolio-specific data, we find that the 6-month/6month and 12-month/12-month momentum strategies are profitable for the industry, size, and B/M portfolios. Like Lewellen, we find the autocovariance component of the momentum profit to be negative, suggesting no return continuations. However, we also find that the autocorrelations calculated from short-term (e.g., monthly) returns are quite different from long-horizon (e.g., annual) autocorrelations. While the first-order autocorrelations of 6- and 12-month returns tend to be negative, the autocorrelations across twelve lags in monthly returns of the industry, size, and B/M portfolios are in general positive. Our results show that these portfolios exhibit return continuations when returns are measured on a monthly basis. Therefore, our finding appears to be consistent with the behavioral models, which suggest positive autocorrelation in stock returns.
\end{abstract}




\section{Introduction}

Among stock return anomalies documented in the finance literature, the profitability of momentum strategies that buy past three- to twelve-month winners and short sell past losers (Jegadeesh and Titman (1993)) remains as a puzzle. Jegadeesh and Titman (2001) document that the profitability of momentum strategies continues in the 1990s, suggesting that their original finding is free of data snooping bias. While momentum strategies tend to generate frequent transactions, Korajczyk and Sadka (2004) find that they remain profitable after accounting for various measures of transaction costs. ${ }^{1}$

Several explanations have been proposed to interpret the momentum in stock returns. For instance, Conrad and Kaul (1998) claim that momentum profits are primarily due to cross-sectional variation in unconditional mean returns. According to Conrad and Kaul's arguments (also see Berk, Green, and Naik (1999)), if realized returns are strongly correlated to expected returns, then past winners (losers) that have higher (lower) returns tend to yield higher (lower) expected returns in the future. Consequently, momentum strategies that buy past winners and short sell past losers will yield positive profits because of the cross-sectional dispersion in unconditional mean returns of stocks. However, Jegadeesh and Titman (2001) suggest that Conrad and Kaul's argument cannot explain the long-term (one- to five-year) return reversal effect documented in the literature. Furthermore, Jegadeesh and Titman (2002) show that Conrad and Kaul's results suffer from small sample biases, and when these biases are corrected for in the tests, the variation in mean returns explains very little of the momentum profits.

Another plausible explanation is that abnormal returns from trading strategies are compensations for risk. However, Fama and French (1996) document that while the Fama and French three-factor model can capture most return anomalies, it cannot explain the short-term momentum. Grundy and Martin (2001) provide further evidence showing that the profitability to momentum strategies cannot be

\footnotetext{
${ }^{1}$ Lesmond, Schill, and Zhou (2004) however suggest that momentum profits reported in prior studies are illusory because of the underestimation of transaction costs. Specifically, they find that momentum strategies require frequent trading in disproportionately loser stocks that tend to be illiquid and hence have larger trading costs. Using various trading cost estimates, they find that costs of executing momentum strategies are much higher than those reported in prior studies, showing that momentum profits are bounded by transaction costs.
} 
explained by exposures to the Fama-French three factors as well as to industry factors. They attribute the major source of the momentum phenomenon to momentum in firm-specified returns. ${ }^{2}$

Momentum can also be attributed to return continuations. Several existing behavioral models (e.g., Barberis, Shleifer, and Vishny (1998), Daniel, Hirshleifer, and Subrahmanyam (1998), and Hong and Stein (1999)) provide a theoretical framework to explain the empirical return anomalies documented in the finance literature. Despite the differences in their underlying assumptions regarding investors' behavioral biases, these behavioral models all suggest that the momentum effect is closely related to positive autocorrelations in stock returns at short horizons. Daniel et al. suggest that investors might not be rational when making decisions based on available information. They suggest that investors suffer from overconfidence that leads them to overweight private information signals and underweight public information signals. They argue that investor's overconfindence, together with biased self-attribution will produce a pattern of stock price changes that exhibits positive (negative) autocorrelation in the short (long) run. Hong and Stein propose that short-term price momentum is a result of underreaction to information when information diffuses gradually across news watchers. They further argue that the initial underreaction will induce trading from momentum traders and hence in the long run prices will reverse to fundamentals when more and more momentum traders enter the market to earn a profit. Similar to Hong and Stein, Barberis et al.'s model is also based on a short-run underreaction. However, unlike Hong and Stien, they obtain the underreaction by assuming that investors exhibit a conservatism bias, meaning that investors are conservative in updating their priors. In short, according to these behavioral models, price momentum is analogical to positive autocorrelation in stock returns, which could arise because of investors' underreaction or continuing overreaction to news.

\footnotetext{
${ }^{2}$ Chordia and Shivakumar (2002) however show that profits to momentum strategies are explained by a set of lagged macroeconomic variables and that firm-specific information explains little of the profits. They suggest the predictability of the set of macroeconomic variables on future stock returns arises because these variables capture time-varying returns. Consequently, they argue that their finding is consistent with the argument of Berk, Green, and Naik (1999) that profitability of momentum strategies represents rewards of bearing risk. However, it is noteworthy that simulation based on the model developed by Berk et al. shows that momentum strategy is not profitable for horizons less than 9 months (e.g., 3- and 6-month), which is inconsistent with the empirical finance
} 
Finally, profits to momentum strategies can arise because of cross-sectional correlations among stocks (Lo and MacKinlay (1990)). Lo and MacKinlay show that momentum profits are negatively related to cross-serial correlations, meaning that negative (positive) cross-serial correlations can lead to profits (losses) for a trading strategy that buys winner stocks and short sells loser stocks. Under this scenario, if winner stocks are negatively cross-serially correlated with loser stocks, then a strategy that buys past winners and short sells past losers can generate profits.

Among these possible sources of momentum profits, the results reported by Lewellen (2002) suggest that momentum is mainly driven by cross-serial covariances in stock returns. Lewellen finds that industry, size, and book-to-market portfolios exhibit similar momentum in returns as that for individual stocks. Using the Lo and MacKinlay (1990) method, Lewellen decomposes the portfolio-based momentum profits into three components: (1) negative of the average first-order cross-serial covariance of annual returns of these portfolios, (2) the average first-order autocovariance in annual returns, and (3) the cross-sectional variation of the mean returns of the portfolios. He finds that both the autocovariances and cross-serial covariances are negative, but the latter is more negative than the former. Thus, he concludes that the profitability of portfolio-based momentum strategies is mainly due to cross-serial correlations among portfolio returns. Lewellen argues that his finding of negative autocovariances is inconsistent with the behavioral models, which imply that stock returns are positively serially correlated.

In this study, we attempt to reconcile the apparently conflicting results between the negative autocorrelations as documented in Lewellen (2002) and the analogy between momentum and positive autocorrelation as suggested by existing behavioral models. While the behavioral models do not provide any guidance about the time length of return horizons that will exhibit positive autocorrelation, Lewellen focuses his analysis on annual horizon. We show that the inconsistency between Lewellen's finding and the behavioral models is due to how one measures the length of return horizons. Conceptually, price momentum should be corresponding to positive autocorrelation. Empirically speaking, the existence of

literature. Thus, under Berk et al.'s framework, it is unclear how well rational asset-pricing models can explain the momentum. 
momentum could go along with negative autocorrelation because the value as well as the sign of serial correlation could vary with return intervals. In other words, autocorrelations may change sign when returns are measured in different horizons. For instance, consider the S\&P 500 total return index (including dividends reinvestments) for the 1941-1999 period—-the sample period that we analyze in this study. The first-order autocorrelation is 0.020 for the monthly returns, while it is -0.100 for the annual returns. Moreover, the average of the first twelve-order autocorrelations in the S\&P 500 monthly returns is a positive number of 0.004 . This finding suggests that the negative autocovariances that Lewellen finds in annual returns could be totally consistent with price momentum, which should be considered more of a phenomenon when pricing adjustments are better evaluated at short, rather than long, intervals. Although the behavioral models do not specify the length of return interval in measuring how stock prices adjust to news, it would be reasonable to expect that the market under- or overreacts to information at a horizon that is much shorter than one year.

To evaluate this issue, we follow Lewellen (2002) and apply the Lo and MacKinlay (1990) momentum decomposition method to industry, size, and book-to-market portfolios for the 1941-1999 sample period. We formulate the portfolio-specific momentum strategies with two intervals, 6 and 12 months. Consistent with Lewellen, we find that both the auto- and cross-serial covariance components of the momentum profit are negative and in general the cross-serial covariances are more negative. As Lewellen indicates, this result implies that momentum is caused by cross-serial covariances, not by positive autocorrelation. However, when the autocorrelations are calculated on monthly returns, most of them are positive. Furthermore, the averages and the cumulative sums of the first six-and twelve-order autocorrelations are mostly positive. Thus, the negative autocovariances associated with momentum as documented in Lewellen and the current study are indeed consistent with the explanation of positive return autocorrelations offered by the behavioral theories. Our results appear to suggest that the relation between price momentum and return autocorrelations is better to be measured by looking at autocorrelations of short-term over various lags rather than autocorrelations of long-term returns. 
The rest of the paper is organized as follows. Section II describes the momentum strategies that we follow in formulating trading rules and discusses the decompositions of momentum profits into various sources. Section III describes the data, presents the portfolio-based momentum profits and the components of the profits, and analyzes the auto- and cross-serial covariances for those portfolios for various return horizons. The conclusion is in the final section.

\section{Momentum Strategies and Sources of Profits}

In this paper, we formulate momentum strategies in the same manner as did in Lewellen (2002). Specifically, we consider a strategy that buys or sells stocks at time $t$, based on their performance at time $t$ -1 , with $k$ horizons between time $t-1$ and $t .^{3}$ The momentum portfolio is constructed with investment weights in stock $i$ determined as:

$$
w_{i, t-1}(k)=(1 / N)\left[R_{i, t-1}(k)-R_{m, t-1}(k)\right],
$$

where $N$ is the number of stocks available, $R_{i, t-1}(k)$ is the return for stock $i$ at time $t-1$, and $R_{m, t-1}(k)=$ $(1 / N) \sum_{i=1}^{N} R_{i, t-1}(k)$ is the return for an equal-weighted portfolio of stock index at time $t-1$, and $k$ is the return interval between time $t-1$ and $t$. Equation (1) shows that the investment weights are determined based on the performance of stocks against the average return of all stocks. That is, the momentum strategy will buy winner stocks and sell loser stocks at time $t-1$ and hold the positions at time $t$ for a horizon of $k$. By construction, the investment weights lead to a zero-cost, arbitrage portfolio since weights sum to zero, i.e., $\sum_{i=1}^{N} w_{i, t-1}(k)=0$. Furthermore, bigger winners and losers will receive greater weights, as can be seen clearly from Equation (1).

The profit from this momentum strategy at time $t, \pi_{t}(k)$, is

$$
\pi_{t}(k)=\sum_{i=1}^{N} w_{i, t-1}(k) R_{i, t}(k)
$$

\footnotetext{
${ }^{3}$ This trading strategy is originally proposed by Lo and MacKinlay (1990) and is employed by Conrad and Kaul (1998) and Jegadeesh and Titman (2002), among others.
} 


$$
\begin{aligned}
& =\frac{1}{N} \sum_{i=1}^{N}\left[R_{i, t-1}(k)-R_{m, t-1}(k)\right] R_{i, t}(k) \\
& =\frac{1}{N} \sum_{i=1}^{N}\left[R_{i, t-1}(k) R_{i, t}(k)\right]-R_{m, t-1}(k) R_{m, t}(k) .
\end{aligned}
$$

Following Lo and MacKinlay (1990) and assuming that unconditional mean returns of individual stocks are constant, we can decompose the momentum profit into various components by taking expectations of Equation (2):

$$
\begin{aligned}
E\left[\pi_{t}(k)\right]= & \frac{1}{N} \sum_{i=1}^{N} E\left[R_{i, t-1}(k) R_{i, t}(k)\right]-E\left[R_{m, t-1}(k) R_{m, t}(k)\right] \\
= & \frac{1}{N} \sum_{i=1}^{N}\left(\operatorname{Cov}\left[R_{i, t-1}(k), R_{i, t}(k)\right]+\mu_{i}^{2}(k)\right)-\left(\operatorname{Cov}\left[R_{m, t-1}(k), R_{m, t}(k)\right]+\mu_{m}^{2}(k)\right) \\
= & -\operatorname{Cov}\left[R_{m, t-1}(k), R_{m, t}(k)\right]+\frac{1}{N} \sum_{i=1}^{N} \operatorname{Cov}\left[R_{i, t-1}(k), R_{i, t}(k)\right] \\
& +\frac{1}{N} \sum_{i=1}^{N}\left[\mu_{i}(k)-\mu_{m}(k)\right]^{2}
\end{aligned}
$$

Equation (3) indicates that the expected profits of momentum strategies come from three sources: (1) the negative of the first-order autocovariance of the $k$-period returns for the equal-weighted market portfolio, (2) the average of the first-order autocovariances of the $k$-period returns of the $N$ stocks, and (3) the variance of the mean returns of the $N$ stocks. Note that if all stock prices follow random walks and hence all the first-order autocovariances equal zero, then the expected momentum profit equals to the crosssectional variation in mean returns. This is what Conrad and Kaul (1998) find, in which they document that momentum is attributable to cross-sectional dispersion in mean returns, not to time-series predictability in returns.

Also note that if we assume every stock has the same mean return, it is easy to see that price momentum (i.e., positive autocovariance) does not necessarily lead to momentum profit. When individual stocks exhibit positive autocovariance, it is reasonable to expect that the equal-weighted market portfolio will also show positive autocovariance. Therefore, in the absence of cross-sectional 
variation in mean returns, whether a momentum strategy is profitable or not would depend on the relative magnitudes between the autocovariances of the market portfolio and those of the individual stocks.

Moreover, as Atchison, Butler, and Simonds (1987) demonstrate, the autocorrelation of a well-diversified portfolio is approximately equal to the average cross-serial covariance between the stocks divided by the average contemporaneous covariance between these stocks. Accordingly, cross-serial covariances between stocks also affect momentum profits.

To see how momentum is affected by cross-serial covariances between individual stocks as well as by autocovariances, note that Equation (3) can be rewritten as follows:

$$
\begin{aligned}
E\left[\pi_{t}(k)\right]= & -\left\{\operatorname{Cov}\left[R_{m, t}-1(k), R_{m, t}(k)\right]-\frac{1}{N^{2}} \sum_{i=1}^{N} \operatorname{Cov}\left[R_{i, t-1}(k), R_{i, t}(k)\right]\right\} \\
& +\frac{N-1}{N^{2}} \sum_{i=1}^{N} \operatorname{Cov}\left[R_{i, t-1}(k), R_{i, t}(k)\right]+\frac{1}{N} \sum_{i=1}^{N}\left[\mu_{i}(k)-\mu_{m}(k)\right]^{2} \\
= & -C_{1}+O_{1}+\sigma^{2}(\mu) .
\end{aligned}
$$

Equation (4) shows that the profitability of a momentum strategy depends not only on the first-order autocovariances of individual stocks, denoted by $O_{1}$, but also on the first-order cross-serial covariances, denoted by $C_{1}$. Momentum profits can also be due to the cross-sectional variation in mean returns of the $N$ stocks, $\sigma^{2}(\mu)$. In summary, the decomposition shows that momentum profits result form three sources. First, stock returns are negatively cross-serially correlated, implying that a stock with a high return today is associated with low returns in the future for other stocks. Second, individual stocks might be positively serially correlated, implying that a stock with a high return today is expected to have high returns in the future. The final source arises because momentum strategy tends to buy stocks with a high mean return and short sell others with a low mean return. The results reported by Lewellen (2002) seem to support the view that momentum is driven by cross-serial covariances between stock returns. Lewellen finds that momentum strategies applied on industry, size, and book-to-market portfolios exhibit similar profits as those for individual stocks. He also finds that the portfolio-based momentum is mainly due to cross-serial covariances between these portfolios because both the average auto- and cross-serial covariances are 
negative, but the latter is more negative than the former. Apparently, Lewellen's finding is inconsistent with the behavioral models, which imply that stock returns are positively serially correlated. Lewellen hypothesizes that the negative cross-serial covariances are a result from the combination of investors' underreactions to firm-specific news and overreactions to macroeconomic news.

Alternatively, Conrad and Kaul (1998) assert that momentum profits are mainly driven by the variation in the mean returns of individual equities, not by the time-series predictability in stock returns. They interpret momentum as variations in risk across assets. Intuitively, if realized returns are strongly correlated to expected returns, then momentum strategies that buy past winners and short sell past losers will yield positive profits because winners (losers) that have higher (lower) realized returns tend to yield higher (lower) expected returns in the future. To see this, consider the case that stock returns are both serially and cross-sectionally uncorrelated (i.e., stock returns are not predictable), then both the first and the second terms in Equation (4) become zero and hence the entire momentum profit is due to the crosssectional variation in mean returns. Thus, in the absence of predictability in stock returns, momentum can still arise as long as there are significant variations in mean returns or time-varying risk premiums. Nevertheless, Jegadeesh and Titman (2002) provide results showing that Conrad and Kaul's results suffer from small sample biases, and when these biases are corrected for in the tests, the variation in mean returns explains very little of the momentum profits. ${ }^{4}$

\section{Data, Momentum Profits, and Autocorrelations}

\section{A. Data}

For the purpose of comparison with Lewellen (2002), we conduct our analyses on portfolios, instead of individual stocks. Monthly return data for industry, size, and book-to-market (B/M) portfolios

\footnotetext{
${ }^{4}$ Since both Conrad and Kaul and Jegadeesh and Titman focus on the contribution of the cross-sectional variation of mean returns to momentum profits, they combine the auto- and cross-serial covariance terms into a time-serial predictability term. Kaul and Jegaseesh's result indeed implies that momentum effect is mainly due to time series predictability in stock returns.
} 
are retrieved from Kenneth French's website. ${ }^{5}$ We examine the period 1941-1999 because Lewellen considers this sample period. These portfolio return data are compiled using all NYSE, AMEX, and Nasdaq common stocks. We use the 12 industry portfolios and 10 size and B/M decile portfolios. Stocks are grouped into industries according to their four-digit SIC codes at the end of June of each year. Size portfolios are constructed using the market equity value at the end of each June and using NYSE breakpoints. B/M portfolios are based on the book-to-market ratio at the end of previous fiscal year. The breakpoints for the $\mathrm{B} / \mathrm{M}$ portfolios are based on those stocks that have data on book value. We conduct the analysis on both value- and equal-weighted portfolios, but only report the results for the valueweighted portfolios given that the results for the equal-weighted portfolios are qualitatively the same.

\section{B. Profits to momentum strategies}

Lewellen (2002) form momentum portfolios based on past 6- and 12-month returns and calculate momentum profits each month up to 18 months after formation. Since our objective is to evaluate the relation between autocovariances and return horizons, we perform the analysis with a ranking period equals to the evaluation period. Table 1 reports the profits to momentum strategies implemented on the different sets of portfolios for two different horizons, with $k$ equals 6 and 12 months. The portfolio-based momentum strategies are profitable except for a 6-month strategy implemented on the B/M portfolios. Indeed, the 6-month strategy applied to the $\mathrm{B} / \mathrm{M}$ portfolios yields a loss, which is somewhat surprising given the finding of Chan, Jegadeesh, and Lakonishok (1996). Chan et al. find that past 6-month winners tend to be glamour stocks with low book-to-market ratios, while past 6-month losers tend to be value stocks with high book-to-market ratios. The $z$-statistics, ${ }^{6}$ which are asymptotically standard normal under the null hypothesis that the "true" profits are zero, show that only the 12-month momentum strategy applied to the industry and size portfolios generates statistically significant profits.

\footnotetext{
${ }^{5}$ The website's address is http://mba.tuck.dartmouth.edu/pages/faculty/ken.french/data_library.html.

${ }^{6}$ The $z$-statistics are corrected for heteroskedasticity and autocorrelations up to eight lags based on the adjustments outlined in Newey and West (1987).
} 
Given our small sample size (59 for the 12-month analysis), the statistical power of the asymptotic statistics tends to be low. To remedy this problem, we perform a bootstrap test. For the bootstrap test, we shuffle (without replacement) the sets of monthly portfolio returns simultaneously so that both auto- and cross-serial correlations in the data are eliminated. ${ }^{7}$ A total of 1,000 replications are implemented. The results from the bootstrap analysis are also provided in Table 1. We focus on using the $p$-value, which is the probability that the 1,000 bootstrap profits are less (larger) than the sample average profit if the sample value is less (larger) than the median of the bootstrap distribution, for statistical inference. The statistical inferences based on the bootstrap analysis are very similar to those from the asymptotic $z$-statistics. Again, the bootstrap $p$-value suggests that the 12-month momentum strategy applied to the industry and size portfolios yields statistically significant profits at the 5 percent level.

Table 1 also reports the momentum profit components based on Equation (4). Consistent with Lewellen (2002), we find the auto- and cross-serial covariances are both negative, except the autocovariance term from the 12-month strategy applied on the size portfolios. Moreover, the cross-serial covariances are in general more negative than the autocovariances. Since momentum profit is contributed by autocovariance positively but by cross-serial covariance negatively, the more negative cross-serial covariance value along with negative autocovariance means that return continuations may not be the source of the momentum profits. Rather, cross-serial covariance appears to dominate the momentum, even though for most of the cases its contribution is partially offset by the autocovariance component. Nevertheless, none of the auto- and cross-serial covariance components is significant at any conventional level based on either asymptotic $z$-statistics or bootstrap estimates.

The cross-sectional variation in the mean returns of these portfolios appears not to affect the momentum significantly for the industry and size portfolios. However, the dispersion in mean returns of

\footnotetext{
${ }^{7}$ Usually bootstrap experiments are done with replacement (e.g., see Conrad and Kaul (1998)). However, as Jegadeesh and Titman (2002) show, bootstrap experiments where returns are drawn with replacement will overestimate the cross-sectional variation in stock mean returns for small samples. Thus, even though shuffling has eliminated any time- and cross-serial dependence in stock returns, bootstrapping with replacement may still generate
} 
the $\mathrm{B} / \mathrm{M}$ portfolios seems to be an important component compared to the time-series predictability because the sum of the auto- and cross-serial covariance components is negative (the 6-month horizon) or one component is almost entirely offset by the other (the 12-month horizon). Similar results have also been documented in Conrad and Kaul (1998), in which they emphasize whether profits to trading strategies are from the time-series predictability in returns (i.e., sum of $-C_{1}$ and $O_{1}$ ) or from the variation in mean returns. Since the two time-series predictability components may offset each other, combining them together in the analysis might overestimate the importance of the variance of mean returns to the profits of momentum strategies.

\section{Auto- and cross-serial correlations}

As Table 1 shows, the averages of the first-order auto- and cross-serial covariance in 6- and 12month returns of the industry, size, and $\mathrm{B} / \mathrm{M}$ portfolios are in general negative. To explore how these portfolios' returns are correlated with their own returns and other portfolios', we calculate the first-order auto- and cross-serial correlations in semi-annual and annual returns. Tables 2,3 , and 4 report the estimates for the industry, size, B/M portfolios, respectively. As can be seen, these portfolios' returns not only negatively correlated with their past returns but also negatively cross-serially correlated with other portfolios' returns. Most of the portfolios' first-order autocorrelations (the entries in diagonals) are negative. For the industry and $\mathrm{B} / \mathrm{M}$ portfolios, the average of the first-order autocorrelations (the last diagonal item) becomes more negative when the horizon increases from 6 months to 12 months, but this is not the case for the size portfolios.

Apparently, these cross-serial correlation matrices are not symmetric. For the size and B/M portfolios, elements below the diagonal are greater than those above it, indicating a lead-lag relation between these portfolios. Specifically, large stocks appear to lead those of smaller stocks, and high B/M stocks lead lower B/M stocks. Unlike the size and B/M portfolios, the industry portfolios do not exhibit

significant profit because of the small sample bias. 
such lead-lag relations. Consistent with the momentum profit decomposition results reported in Table 1, most the averages of these cross-serial covariances are negative.

As can be seen in Table 2, the first-order cross-serial correlations in returns of the industry portfolios tend to be larger (in absolute value) than the autocorrelations. For instance, at a 6-month horizon, the average of the cross-serial correlations is -0.036 (calculated from the twenty-four average cross-serial correlation figures in Table 2), which is much smaller than that of the autocorrelations (i.e., -0.003). Similar results also hold for the size portfolios (see Table 3). This finding of larger (in absolute value) cross-serial correlations is consistent with Table 1, as well as with Lewellen's (2002) claim that the main contribution to the momentum profit comes from negative cross-serial correlations between securities. The $\mathrm{B} / \mathrm{M}$ portfolios, however, do not show that the cross-serial covariance dominates the momentum. The average of the semi-annual cross-serial correlations $(-0.024$, calculated from the twenty average figures in Table 4) of the $\mathrm{B} / \mathrm{M}$ portfolios is slightly larger than the semi-annual autocorrelation $(-0.027)$, whereas the average annual cross-serial correlation $(-0.089)$ is slightly less than the annual autocorrelation $(-0.082)$.

\section{Autocorrelations and the return horizon}

The evidence presented so far supports the idea that negative cross-serial correlations between securities are the driving force of the intermediate-horizon momentum. Return continuations (positive autocorrelations), as behavioral models (e.g., Barberis et al. (1998), Daniel et al. (1998), and Hong and Stein (1999)) suggest, cannot explain the momentum because negative return autocorrelations are found at intermediate horizons, such as 6- and 12-months. It is noteworthy, however, that short-run momentum (long-term reversals) is associated with positive (negative) autocorrelation of short-term returns separated by short (long) lags. For instance, Hong and Stein's (1999) Proposition 2 indicates that a momentum strategy will be profitable for a horizon $k$ if the cumulative autocovariances of returns up to $k-1$ is not zero (p. 2154). According to Hong and Stein, when cumulative autocorrelations of short-horizon returns 
are positive up to a certain lag order, price momentum will arise. In other words, return continuations that the behavioral models refer to are a feature that is more to do with short-horizon returns than with longhorizon returns. On the other hand, the Lo and MacKinlay (1990) decomposition method allows us to evaluate only the first-order autocorrelation of long-horizon returns, not the autocorrelations of short-term returns separated by short lags. Therefore, one possible explanation that the momentum decomposition result does not show return continuations as predicted by the behavioral models is due to its failure of capturing the autocorrelations of short-horizon returns. Moreover, intuitively it seems to be more appropriate to rely on short-horizon returns, rather than long-horizon returns, if one wants to measures investors' over- or underreaction to information arriving to the market. In short, our view is that, even though long-horizon stock returns are negatively serially correlated, short-horizon stock returns could show positive autocorrelations.

To investigate this possibility, we calculate autocorrelations of monthly returns for first order to twelfth order. Table 5 provides estimates of these monthly autocorrelations. Most autocorrelations are positive and not significantly different from zero, except for the first-order autocorrelations. Unlike annual autocorrelations, all the first-order monthly autocorrelations are positive, suggesting that return continuations do exist. However, after a price continuation for two consecutive months, stock prices in general take a correction at the opposite direction in the following month, as negative second-order autocorrelations imply. Consistent with the literature, small-firm portfolios display larger first-order autocorrelations than those of large-firm portfolios. The larger autocorrelations for small-firm portfolios likely are induced by much slow pricing adjustments to information by small firms compared to larger firms. ${ }^{8}$

To get a clear picture of autocorrelation patterns, we calculate the average of the autocorrelations for lags 1-6 and 1-12 separately as well as their cumulative sum. The average autocorrelation and the cumulative autocorrelation over 6 months are uniformly positive for the industry, size, and B/M

\footnotetext{
${ }^{8}$ Hong, Lim, and Stein (2000) find that momentum strategies become less profitable when extreme small stocks are excluded from the analysis. Their finding suggests that small stocks exhibit more pronounced momentum and
} 
portfolios. Similar to the first-order autocorrelation, the average and the cumulative autocorrelations over 6 months decline as firm sizes increase. The majority of the average and the cumulative autocorrelation over 12 months are also positive. Overall, the results in Table 5 indicate that autocorrelations across 12 months are in general positive. Consequently, our findings support the behavioral models' argument that momentum is analogous to positive medium-term autocorrelations in stock returns.

\section{Conclusions}

In this study we examine Lewellen's (2002) claim that momentum is not due to positive autocorrelation as suggested by existing behavioral modes. All the behavioral models have corresponded momentum with positive autocorrelation. Using portfolio-specific data, we find that the 6-month/6month and 12-month/12-month momentum strategies are profitable for the industry, size, and B/M portfolios. Consistent with Lewellen, we find that the autocovariance components of the momentum profits are in general negative. This finding appears not to support the correspondence between momentum and positive autocorrelations in stock returns. The autocorrelation analysis also shows that both the first-order auto- and cross-serial correlations in 6- and 12-month portfolio returns are negative.

We argue that the analogous between positive autocorrelation and momentum is better evaluated by using short-term returns. That is, momentum arises because short-horizon returns are positively serially correlated over short lags. The monthly autocorrelations seem to support this conjecture. Specifically, most autocorrelations across twelve lags in monthly returns of the industry, size, and B/M portfolios are positive. Our results therefore imply that these portfolios exhibit return continuations, which appear to be consistent with the behavioral models.

positive autocorrelations because they adjust more slowly to news than larger stocks. 


\section{REFERENCES}

Atchison, M., K. Butler, and R. Simonds, 1987, Nonsynchronous security trading and market index autocorrelation, Journal of Finance 42, 533-553.

Barberis, Nicholas, Andrei Shleifer, and Robert Vishny, 1998, A model of investor sentiment, Journal of Financial Economics 49, 307-343.

Berk, Jonathan B., Richard C. Green, and Vasant Naik, 1999, Optimal investment, growth options, and security returns, Journal of Finance 54, 1553-1608.

Chan, Louis K. C., Narasimhan Jegadeesh, and Josef Lakonishok, 1996, Momentum strategies, Journal of Finance 51, 1681-1713.

Chordia, Tarun, and Lakshmanan Shivakumar, 2002, Momentum, business cycles, and time-varying expected returns, Journal of Finance 57, 985-1019.

Conrad, Jennifer, and Gautam Kaul, 1998, An anatomy of trading strategies, Review of Financial Studies $11,489-519$.

Daniel, Kent D., David Hirshleifer, and Avanidhar Subrahmanyam, 1998, Investor psychology and security market under- and overreactions, Journal of Finance 53, 1839-1885.

Fama, Eugene F, and Kenneth R. French, 1996, Multifactor explanations of Asset Pricing anomalies, Journal of Finance 51, 55-84.

Grundy, Bruce D., and J. Spencer Martin, 2001, Understanding the nature of the risks and the source of the rewards to momentum investing, Review of Financial Studies 14, 29-78.

Hong, Harrison, and Jeremy C. Stein, 1999, A unified theory of underreaction, momentum trading and overreaction in asset markets, Journal of Finance 54, 2143-2184.

Hong, Harrision, Terence Lim, and Jeremy C. Stein, 2000, Bad news travels slowly: Size, analyst coverage, and the profitability of momentum strategies, Journal of Finance 55, 265-295.

Jegadeesh, Narasimhan, and Sheridan Titman, 1993, Returns to buying winners and selling losers: Implications for stock market efficiency, Journal of Finance 48, 65-91. 
Jegadeesh, Narasimhan, and Sheridan Titman, 2001, Profitability of momentum strategies: An evaluation of alternative explanations, Journal of Finance 56, 699-720.

Jegadeesh, Narasimhan, and Sheridan Titman, 2002, Cross-sectional and time-series determinants of momentum returns, Review of Financial Studies 15, 143-157.

Korajczyk, Robert, and Ronnie Sadka, 2004, Are momentum profits robust to trading costs?, Journal of Finance 59, 1039-1082.

Lewellen, Jonathan, 2002, Momentum and autocorrelation in stock returns, Review of Financial Studies $15,533-563$.

Lesmond, David A., Michael J. Schill, and Chunsheng Zhou, 2004, The illusory nature of momentum profits, Journal of Financial Economics 71, 349-380.

Lo, Andrew W., and A. Craig MacKinlay, 1990, When are contrarian profits due to stock market overreaction?, Review of Financial Studies 3, 175-205.

Newey, Whitney K., and Kenneth D. West, 1987, A simple, positive semi-definite heteroskedasticity and autocorrelation consistent covariance matrix, Econometrica 55, 703-708. 
Table 1

Profits to Momentum Strategies and Auto- and Cross-serial Covariances

This table contains the decomposition of average profits of momentum strategies that long past winner portfolios and short past loser portfolios for the period 1941-1999. Expected profit is given by $\mathrm{E}\left[\pi_{\mathrm{t}}(k)\right]=-C_{1}+O_{1}+\sigma^{2}(\mu)$, where $C_{1}$ mainly depends on the average first-order cross-serial covariance of the portfolio returns, $O_{1}$ depends on the average first-order autocovariance, and $\sigma^{2}(\mu)$ is the cross-sectional variance of the mean returns. The numbers in parentheses are $z$-statistics that are asymptotically $N(0,1)$ under the null hypothesis that the relevant parameter is zero, and are robust to autocorrelation and heteroskedasticity. The p-value reports the probability that the 1,000 bootstrap average profits from the bootstrap distribution are less (larger) than the sample average profit if the sample value is less (larger) than the median of the bootstrap distribution. The p-value reports the probability that the 1,000 bootstrap average profits from the bootstrap distribution are less (larger) than the sample average profit if the sample value is less (larger) than the median of the bootstrap distribution. All profit estimates are multiplied by 1,000.

\begin{tabular}{|c|c|c|c|c|}
\hline Horizon & $\mathrm{E}\left[\pi_{\mathrm{t}}(k)\right]$ & $C_{1}$ & $O_{1}$ & $\sigma^{2}(\mu)$ \\
\hline \multicolumn{5}{|c|}{12 industry value-weighted portfolios } \\
\hline \multirow[t]{3}{*}{6 -month } & 0.375 & -0.564 & -0.218 & 0.029 \\
\hline & $(1.41)$ & $(-0.42)$ & $(-0.14)$ & \\
\hline & $p=.011$ & $p=.333$ & $p=.473$ & \\
\hline \multirow[t]{3}{*}{$12-$ month } & 1.027 & -1.485 & -0.574 & 0.116 \\
\hline & $(1.86)$ & $(-0.48)$ & $(-0.17)$ & \\
\hline & $p=.024$ & $p=.336$ & $p=.500$ & \\
\hline \multicolumn{5}{|c|}{10 size value-weighted portfolios } \\
\hline \multirow[t]{3}{*}{6 -month } & 0.068 & -0.698 & -0.648 & 0.017 \\
\hline & $(0.50)$ & $(-0.36)$ & $(-0.33)$ & \\
\hline & $p=.333$ & $p=.384$ & $p=.403$ & \\
\hline \multirow[t]{3}{*}{12 -month } & 1.553 & -0.739 & 0.745 & 0.069 \\
\hline & $(2.29)$ & $(-0.14)$ & $(0.13)$ & \\
\hline & $p=.001$ & $p=.471$ & $p=.402$ & \\
\hline \multicolumn{5}{|c|}{$10 \mathrm{~B} / \mathrm{M}$ value-weighted portfolios } \\
\hline \multirow[t]{3}{*}{6 -month } & -0.028 & -0.279 & -0.396 & 0.089 \\
\hline & $(-0.24)$ & $(-0.20)$ & $(-0.28)$ & \\
\hline & $p=.219$ & $p=.450$ & $p=.421$ & \\
\hline \multirow[t]{3}{*}{12 -month } & 0.540 & -1.829 & -1.644 & 0.354 \\
\hline & $(1.07)$ & $(-0.49)$ & $(-0.40)$ & \\
\hline & $p=.229$ & $p=.319$ & $p=.355$ & \\
\hline
\end{tabular}


Table 2

Autocorrelations and Cross-serial Correlations of Industry Portfolios

This table reports first-order autocorrelations (diagonals) and cross-serial correlations (off diagonals) for lag 1 of the returns of 12 industry portfolios. The industry portfolios are constructed based on four-digit SIC codes. Ind1 is for consumer non-durables, Ind2 is for consumer durables, Ind3 is for manufacturing, Ind4 is for energy, Ind5 is for chemicals, Ind6 is for business equipment, Ind7 is for telecommunication, Ind8 is for utilities, Ind9 is for wholesale and retail, Ind10 is for healthcare, Indl1 is for finance, and Ind12 is for all other industries. The entry in the ith row and jth column is the correlation between $R_{i, t-1}$ and $R_{j, t}$. Standard error is calculated as $1 / \sqrt{ } T$, where $T$ is the number of non-overlapping returns. T is 118 for semi-annual returns, and is 59 for annual returns. Bold denotes estimates are significantly different from zero at the $10 \%$ level.

\begin{tabular}{|c|c|c|c|c|c|c|c|c|c|c|c|c|c|}
\hline Portfolio & Ind1 & Ind2 & Ind3 & Ind 4 & Ind5 & Ind6 & Ind7 & Ind8 & Ind9 & Ind10 & Ind11 & Ind12 & Average \\
\hline \multicolumn{14}{|c|}{ Semi-annual returns } \\
\hline Ind1 & -.000 & -.060 & -.057 & -.073 & -.031 & -.105 & .079 & .044 & -.032 & -.008 & -.052 & -.069 & -.033 \\
\hline Ind2 & -.085 & -.042 & -.032 & -.069 & -.019 & .004 & .078 & .001 & -.051 & -.034 & -.094 & -.025 & -.030 \\
\hline Ind3 & -.120 & -.098 & -.065 & -.079 & -.049 & -.082 & -.027 & -.081 & -.115 & -.044 & -.148 & -.104 & -.086 \\
\hline Ind 4 & -.044 & .018 & .028 & -.015 & .050 & -.006 & -.079 & -.093 & .000 & -.003 & -.087 & -.003 & -.020 \\
\hline Ind5 & -.126 & -.069 & -.079 & -.077 & -.042 & -.111 & -.027 & -.069 & -.111 & -.063 & -.160 & -.134 & -.093 \\
\hline Ind 6 & -.095 & -.079 & -.037 & -.115 & -.079 & -.021 & .016 & -.078 & -.086 & -.065 & -.076 & -.064 & -.069 \\
\hline Ind7 & -.041 & .039 & -.028 & -.066 & -.009 & .026 & .196 & .014 & .027 & -.021 & -.096 & -.021 & -.016 \\
\hline Ind8 & .165 & .153 & .163 & .074 & .146 & .103 & .192 & .140 & .189 & .108 & .077 & .142 & .137 \\
\hline Ind9 & -.114 & -.116 & -.125 & -.160 & -.098 & -.107 & .003 & -.025 & -.116 & -.058 & -.132 & -.147 & -.098 \\
\hline Ind10 & -.104 & -.118 & -.092 & -.155 & -.068 & -.109 & -.046 & -.067 & -.115 & -.014 & -.150 & -.153 & -.107 \\
\hline Ind1 1 & .024 & .050 & .008 & -.033 & .050 & -.010 & .103 & .037 & .059 & .033 & -.007 & .002 & .029 \\
\hline Ind12 & -.063 & -.036 & -.042 & -.090 & -.002 & -.044 & .010 & -.027 & -.044 & -.021 & -.103 & -.050 & -.042 \\
\hline Average & -.055 & -.029 & -.027 & -.077 & -.010 & -.040 & .027 & -.031 & -.025 & -.016 & -.093 & -.052 & -.003 \\
\hline \multicolumn{14}{|c|}{ Annual returns } \\
\hline Ind1 & .037 & -.031 & .013 & .039 & .018 & -.086 & .113 & .047 & .019 & .065 & .049 & -.000 & .022 \\
\hline Ind2 & -.178 & -.090 & -.030 & .040 & -.076 & -.054 & .065 & -.009 & -.135 & -.110 & -.078 & -.072 & -.058 \\
\hline Ind3 & -.201 & -.242 & -.107 & .007 & -.149 & -.205 & -.096 & -.090 & -.265 & -.076 & -.112 & -.192 & -.147 \\
\hline Ind4 & -.266 & -.270 & -.211 & -.054 & -.191 & -.263 & -.181 & -.147 & -.297 & -.048 & -.161 & -.185 & -.202 \\
\hline Ind5 & -.165 & -.112 & -.039 & .082 & -.036 & -.136 & .024 & -.051 & -.194 & -.055 & -.094 & -.126 & -.079 \\
\hline Ind6 & -.108 & -.072 & .019 & .104 & -.054 & .019 & .098 & .006 & -.084 & -.069 & .069 & -.022 & -.010 \\
\hline Ind7 & -.115 & -.005 & -.116 & -.122 & -.041 & .021 & .069 & -.097 & -.043 & -.078 & -.196 & -.144 & -.085 \\
\hline Ind8 & -.029 & -.036 & .044 & -.031 & .039 & -.032 & .018 & -.031 & -.020 & .007 & -.098 & -.014 & -.014 \\
\hline Ind9 & -.075 & .022 & .041 & .049 & -.003 & -.034 & .093 & .011 & -.022 & -.028 & .016 & -.007 & .008 \\
\hline Ind 10 & -.079 & -.088 & -.039 & .018 & -.025 & -.076 & -.021 & -.108 & -.091 & -.002 & -.113 & -.103 & -.066 \\
\hline Ind11 & -.164 & -.081 & -.078 & -.040 & -.036 & -.101 & -.011 & -.055 & -.105 & -.013 & -.068 & -.111 & -.072 \\
\hline Ind12 & -.114 & -.152 & -.042 & -.001 & -.101 & -.113 & -.079 & -.038 & -.141 & .000 & -.077 & -.077 & -.078 \\
\hline Average & -.121 & -.097 & -.040 & .013 & -.056 & -.098 & .002 & -.048 & -.123 & -.037 & -.072 & -.089 & -.030 \\
\hline
\end{tabular}


Table 3

Autocorrelations and Cross-serial Correlations of Size Portfolios

This table reports first-order autocorrelations (diagonals) and cross-serial correlations (off diagonals) for lag 1 of the returns of 10 size-sorted decile portfolios. The entry in the ith row and jth column is the correlation between

$R_{i, t-1}$ and $R_{j, t}$. Standard error is calculated as $1 / \sqrt{ } T$, where $T$ is the number of non-overlapping returns. $T$ is 118 for semi-annual returns, and is 59 for annual returns. Bold denotes estimates are significantly different from zero at the 10\% level.

\begin{tabular}{|c|c|c|c|c|c|c|c|c|c|c|c|}
\hline Portfolio & Small & 2 & 3 & 4 & 5 & 6 & 7 & 8 & 9 & Large & Average \\
\hline \multicolumn{12}{|c|}{ Semi-annual returns } \\
\hline Small & -.015 & -.014 & -.035 & -.018 & -.052 & -.042 & -.054 & -.083 & -.079 & -.072 & -.050 \\
\hline 2 & -.004 & -.009 & -.039 & -.018 & -.054 & -.049 & -.057 & -.095 & -.086 & -.061 & -.051 \\
\hline 3 & -.012 & -.019 & -.056 & -.038 & -.066 & -.063 & -.070 & -.110 & -.095 & -.073 & -.061 \\
\hline 4 & -.003 & -.008 & -.045 & -.027 & -.058 & -.054 & -.064 & -.101 & -.086 & -.064 & -.054 \\
\hline 5 & .009 & .001 & -.038 & -.016 & -.048 & -.049 & -.057 & -.092 & -.082 & -.048 & -.041 \\
\hline 6 & .020 & .009 & -.030 & -.010 & -.043 & -.043 & -.052 & -.094 & -.077 & -.045 & -.036 \\
\hline 7 & .034 & .027 & -.014 & .008 & -.024 & -.024 & -.031 & -.072 & -.054 & -.012 & -.015 \\
\hline 8 & .029 & .024 & -.021 & .001 & -.028 & -.036 & -.039 & -.079 & -.064 & -.021 & -.017 \\
\hline 9 & .029 & .031 & -.010 & .013 & -.016 & -.016 & -.019 & -.052 & -.032 & .022 & -.002 \\
\hline Large & -.043 & -.038 & -.082 & -.060 & -.080 & -.076 & -.074 & -.111 & -.071 & -.012 & -.071 \\
\hline Average & .004 & .001 & -.035 & -.015 & -.047 & -.045 & -.054 & -.090 & -.077 & -.042 & -.035 \\
\hline \multicolumn{12}{|c|}{ Annual returns } \\
\hline Small & .132 & .119 & .104 & .075 & .085 & .078 & .043 & .002 & .011 & -.043 & .053 \\
\hline 2 & .072 & .071 & .055 & .028 & .045 & .041 & .012 & -.027 & -.019 & -.049 & .018 \\
\hline 3 & .024 & .025 & .013 & -.011 & .007 & .006 & -.027 & -.063 & -.054 & -.076 & -.019 \\
\hline 4 & .010 & .021 & .005 & -.019 & .007 & .008 & -.021 & -.054 & -.044 & -.053 & -.013 \\
\hline 5 & -.015 & .000 & -.012 & -.029 & -.016 & -.005 & -.038 & -.071 & -.065 & -.062 & -.033 \\
\hline 6 & -.042 & -.033 & -.041 & -.061 & -.044 & -.034 & -.065 & -.097 & -.087 & -.085 & -.062 \\
\hline 7 & -.061 & -.041 & -.053 & -.063 & -.046 & -.038 & -.059 & -.091 & -.077 & -.057 & -.059 \\
\hline 8 & -.073 & -.054 & -.065 & -.075 & -.059 & -.043 & -.072 & -.103 & -.096 & -.068 & -.067 \\
\hline 9 & -.095 & -.059 & -.077 & -.083 & -.067 & -.053 & -.059 & -.090 & -.076 & -.022 & -.067 \\
\hline Large & -.211 & -.166 & -.190 & -.192 & -.156 & -.158 & -.138 & -.155 & -.131 & -.047 & -.166 \\
\hline Average & -.026 & -.021 & -.030 & -.046 & -.025 & -.018 & -.041 & -.072 & -.062 & -.057 & -.014 \\
\hline
\end{tabular}


Table 4

Autocorrelations and Cross-serial Correlations of Book-to-Market Portfolios

This table reports first-order autocorrelations (diagonals) and cross-serial correlations (off diagonals) for lag 1 of the returns of 10 book-to-market decile portfolios. The entry in the ith row and jth column is the correlation between $R_{i, t-1}$ and $R_{j, t}$. Standard error is calculated as $1 / \sqrt{ } T$, where $T$ is the number of non-overlapping returns. $T$ is 118 for semi-annual returns, and is 59 for annual returns. Bold denotes estimates are significantly different from zero at the $10 \%$ level.

\begin{tabular}{|c|c|c|c|c|c|c|c|c|c|c|c|}
\hline Portfolio & Low & 2 & 3 & 4 & 5 & 6 & 7 & 8 & 9 & High & Average \\
\hline \multicolumn{12}{|c|}{ Semi-annual returns } \\
\hline Low & -.081 & -.156 & -.139 & -.132 & -.080 & -.085 & -.072 & -.077 & -.107 & -.089 & -.104 \\
\hline 2 & -.045 & -.102 & -.068 & -.067 & -.038 & -.043 & -.043 & -.047 & -.067 & -.063 & -.053 \\
\hline 3 & .003 & -.058 & -.021 & -.029 & -.004 & -.020 & -.022 & -.009 & -.042 & -.059 & -.027 \\
\hline 4 & -.046 & -.087 & -.072 & -.044 & -.033 & -.041 & -.031 & -.014 & -.036 & -.035 & -.044 \\
\hline 5 & .058 & -.009 & .010 & .029 & .035 & .018 & .010 & .046 & .013 & -.005 & .019 \\
\hline 6 & .011 & -.035 & -.015 & .008 & .016 & .009 & .009 & .034 & -.004 & .004 & .003 \\
\hline 7 & .040 & -.006 & .006 & .037 & .019 & .028 & .015 & .036 & .005 & .003 & .019 \\
\hline 8 & .047 & .002 & .017 & .020 & .020 & .031 & .006 & .036 & .010 & -.005 & .016 \\
\hline 9 & -.009 & -.053 & -.024 & -.018 & -.005 & -.020 & -.043 & -.008 & -.038 & -.045 & -.025 \\
\hline High & -.014 & -.048 & -.032 & -.034 & -.030 & -.031 & -.061 & -.038 & -.060 & -.075 & -.039 \\
\hline Average & -.004 & -.050 & -.035 & -.021 & -.015 & -.018 & -.027 & -.009 & -.032 & -.033 & -.027 \\
\hline \multicolumn{12}{|c|}{ Annual returns } \\
\hline Low & -.062 & -.127 & -.150 & -.084 & -.081 & -.063 & -.120 & -.109 & -.122 & -.128 & -.109 \\
\hline 2 & -.050 & -.080 & -.101 & -.022 & -.025 & -.011 & -.101 & -.110 & -.086 & -.078 & -.065 \\
\hline 3 & -.016 & -.043 & -.069 & .010 & -.019 & -.013 & -.092 & -.098 & -.071 & -.102 & -.049 \\
\hline 4 & -.059 & -.068 & -.106 & -.034 & -.043 & -.030 & -.080 & -.116 & -.065 & -.073 & -.071 \\
\hline 5 & -.034 & -.070 & -.106 & -.018 & -.037 & -.027 & -.082 & -.086 & -.052 & -.069 & -.060 \\
\hline 6 & -.117 & -.158 & -.171 & -.103 & -.100 & -.102 & -.147 & -.160 & -.127 & -.144 & -.136 \\
\hline 7 & -.111 & -.121 & -.146 & -.090 & -.098 & -.080 & -.149 & -.154 & -.116 & -.112 & -.114 \\
\hline 8 & -.120 & -.154 & -.170 & -.111 & -.064 & -.073 & -.159 & -.155 & -.131 & -.139 & -.125 \\
\hline 9 & -.056 & -.066 & -.106 & -.049 & -.019 & -.010 & -.090 & -.099 & -.049 & -.048 & -.060 \\
\hline High & -.134 & -.133 & -.147 & -.085 & -.036 & -.031 & -.119 & -.132 & -.091 & -.080 & -.101 \\
\hline Average & -.076 & -.104 & -.134 & -.061 & -.054 & -.038 & -.110 & -.118 & -.096 & -.099 & -.082 \\
\hline
\end{tabular}


Table 5

Autocorrelations in Industry, Size, and B/M Portfolios

This table reports autocorrelations $\left(\rho_{\mathrm{k}}\right)$ for lags 1-12 of the monthly returns for value-weighted industry, size, and B/M portfolios. Ind1 is for consumer non-durables, Ind2 is for consumer durables, Ind3 is for manufacturing, Ind4 is for energy, Ind5 is for chemicals, Ind6 is for business equipment, Ind7 is for telecommunication, Ind8 is for utilities, Ind9 is for wholesale and retail, Ind10 is for healthcare, Ind11 is for finance, and Indl2 is for all other industries. $\rho_{1-6}$ and $\rho_{1-12}$ are the average autocorrelation for lags of $1-6$ and of $1-12$, respectively. $\rho_{1-6, s}$ and $\rho_{1-12, s}$ report the cumulative autocorrelation for lags of $1-6$ and of $1-12$, respectively. $Q(k)$ is the Ljung-Box $Q$ statistic for $k$ order autocorrelation. Bold denotes estimates are significantly different from zero at the $10 \%$ level.

\begin{tabular}{|c|c|c|c|c|c|c|c|c|c|c|c|c|c|c|c|}
\hline Portfolio & $\rho_{1}$ & $\rho_{2}$ & $\rho_{3}$ & $\rho_{4}$ & $\rho_{5}$ & $\rho_{6}$ & $\rho_{8}$ & $\rho_{10}$ & $\rho_{12}$ & $\rho_{1-6}$ & $\rho_{1-12}$ & $\rho_{1-6, \mathrm{~s}}$ & $\rho_{1-12, \mathrm{~s}}$ & $Q(6)$ & $Q(12)$ \\
\hline \multicolumn{16}{|c|}{12 industry value-weighted portfolios } \\
\hline Ind 1 & .132 & -.015 & -.010 & .006 & .079 & -.036 & -.003 & -.015 & .042 & .026 & .018 & .156 & .210 & 18.74 & 19.74 \\
\hline Ind2 & .083 & .028 & -.006 & -.012 & .037 & -.040 & -.014 & .018 & .027 & .015 & .012 & .090 & .146 & 7.75 & 10.33 \\
\hline Ind3 & .083 & -.019 & -.025 & -.017 & .020 & -.036 & -.038 & -.018 & .001 & .001 & -.014 & .006 & -.044 & 7.07 & 8.43 \\
\hline Ind 4 & .027 & -.032 & .012 & .039 & .048 & -.034 & -.076 & -.031 & .026 & .010 & -.001 & .060 & -.014 & 4.91 & 10.87 \\
\hline Ind5 & .027 & -.049 & .021 & -.002 & .055 & -.035 & -.014 & -.021 & .021 & .003 & .000 & .017 & .002 & 5.51 & 6.47 \\
\hline Ind6 & .077 & -.015 & .021 & -.012 & -.005 & -.021 & -.024 & -.023 & .037 & .008 & .007 & .045 & .082 & 5.17 & 7.94 \\
\hline Ind7 & .052 & .003 & .087 & -.007 & .077 & -.007 & .019 & .007 & .064 & .034 & .022 & .205 & .267 & 11.67 & 18.01 \\
\hline Ind8 & .086 & -.050 & .017 & .079 & .155 & -.015 & -.024 & -.004 & .018 & .045 & .022 & .272 & .268 & 29.07 & 29.84 \\
\hline Ind9 & .165 & .005 & -.040 & -.025 & .044 & -.066 & -.014 & .044 & .026 & .014 & .012 & .083 & .143 & 25.42 & 32.25 \\
\hline Ind10 & .052 & .003 & -.023 & .032 & .038 & -.054 & .032 & -.026 & .055 & .008 & .012 & .048 & .146 & 6.15 & 10.63 \\
\hline Ind11 & .123 & -.018 & -.018 & .022 & .063 & -.011 & -.101 & -.036 & .039 & .027 & .005 & .161 & .058 & 14.53 & 23.88 \\
\hline Ind12 & .121 & .010 & -.027 & -.008 & .032 & -.026 & -.105 & -.014 & .014 & .017 & -.001 & .102 & -.008 & 12.32 & 21.06 \\
\hline Average & .086 & -.012 & .001 & .008 & .054 & -.032 & -.030 & -.010 & .031 & .017 & .009 & .104 & .105 & & \\
\hline \multicolumn{16}{|c|}{10 size value-weighted portfolios } \\
\hline Small & .220 & -.001 & -.001 & .034 & .005 & .032 & -.041 & -.032 & .122 & .048 & .028 & .289 & .339 & 36.02 & 50.32 \\
\hline 2 & .194 & .020 & -.014 & -.003 & .002 & .007 & -.058 & -.031 & .070 & .034 & .019 & .206 & .227 & 27.09 & 34.16 \\
\hline 3 & .164 & -.010 & -.024 & -.012 & -.004 & .002 & -.072 & -.023 & .046 & .019 & .007 & .116 & .084 & 19.69 & 25.89 \\
\hline 4 & .177 & -.007 & -.026 & -.025 & .005 & .005 & -.070 & -.030 & .033 & .022 & .009 & .129 & .105 & 23.38 & 28.90 \\
\hline 5 & .145 & -.005 & -.012 & -.013 & .010 & -.002 & -.080 & -.030 & .019 & .021 & .004 & .123 & .044 & 15.35 & 21.42 \\
\hline 6 & .144 & -.012 & -.023 & -.023 & .027 & -.007 & -.094 & -.023 & .019 & .018 & .002 & .106 & .025 & 16.08 & 23.45 \\
\hline 7 & .130 & -.006 & -.017 & -.006 & .034 & -.012 & -.078 & -.021 & .001 & .021 & .002 & .123 & .021 & 13.18 & 18.08 \\
\hline 8 & .096 & -.005 & -.028 & -.025 & .049 & -.027 & -.077 & -.035 & .002 & .010 & -.008 & .060 & -.092 & 9.84 & 15.56 \\
\hline 9 & .083 & -.019 & -.020 & .005 & .052 & -.031 & -.059 & -.019 & .005 & .012 & -.004 & .070 & -.044 & 8.05 & 11.30 \\
\hline Large & .020 & -.014 & .014 & .017 & .093 & -.057 & -.010 & -.021 & .054 & .012 & .007 & .073 & .082 & 9.26 & 11.82 \\
\hline Average & .137 & -.006 & -.015 & -.005 & .027 & -.009 & -.064 & -.027 & .037 & .022 & .007 & .130 & .079 & & \\
\hline
\end{tabular}


$10 \mathrm{~B} / \mathrm{M}$ value-weighted portfolios

\begin{tabular}{|c|c|c|c|c|c|c|c|c|c|c|c|c|c|c|c|}
\hline Low & .094 & -.018 & .005 & -.005 & .046 & -.047 & -.010 & -.031 & .044 & .013 & .005 & .075 & .062 & 9.68 & 12.02 \\
\hline 2 & .067 & -.041 & -.022 & -.001 & .044 & -.038 & -.068 & -.013 & .014 & .002 & -.005 & .009 & -.061 & 7.18 & 10.83 \\
\hline 3 & .071 & -.044 & -.001 & .004 & .062 & -.036 & -.063 & -.008 & .004 & .009 & -.003 & .056 & -.037 & 8.57 & 12.05 \\
\hline 4 & .083 & -.034 & -.026 & .025 & .081 & -.040 & -.067 & .017 & -.002 & .015 & .002 & .089 & .026 & 12.42 & 16.02 \\
\hline 5 & .064 & -.031 & -.024 & .020 & .102 & -.010 & -.084 & -.013 & .010 & .020 & .003 & .121 & .037 & 11.77 & 17.09 \\
\hline 6 & .047 & -.017 & .027 & .001 & .074 & -.027 & -.103 & -.019 & .008 & .018 & .000 & .105 & .003 & 6.73 & 14.64 \\
\hline 7 & .023 & .039 & .033 & -.020 & .104 & -.034 & -.031 & -.045 & .000 & .024 & .004 & .145 & .043 & 11.03 & 13.39 \\
\hline 8 & .039 & .029 & .007 & -.002 & .072 & .005 & -.079 & -.035 & .058 & .025 & .008 & .150 & .096 & 5.38 & 13.69 \\
\hline 9 & .080 & .002 & .018 & .007 & .038 & -.008 & -.060 & -.054 & .058 & .023 & .007 & .137 & .078 & 5.92 & 13.36 \\
\hline High & .110 & .018 & -.042 & .006 & .011 & .010 & -.079 & -.061 & .062 & .019 & .003 & .113 & .031 & 10.24 & 21.30 \\
\hline Average & .068 & -.010 & -.003 & .004 & .063 & -.023 & -.064 & -.026 & .026 & .017 & .002 & .100 & .028 & & \\
\hline
\end{tabular}

